\title{
AVALIAÇÃO DA PRODUÇÃO DE BIOMASSA DO FUSTE DE UM CLONE HÍBRIDO DE EUCALIPTO SOB DIFERENTES ESPAÇAMENTOS
}

\author{
EVALUATION OF THE BIOMASS PRODUCTION OF THE MAIN STEM OF A HYBRID \\ EUCALYPTUS CLONE UNDER DIFFERENT SPACINGS
}

\author{
Maria Dolores dos Santos Barzotto Ribeiro ${ }^{1}$ Luiz Alberto Blanco Jorge ${ }^{2}$ Martha Maria Mischan ${ }^{3}$ \\ André Luis dos Santos ${ }^{4}$ Adriano Wagner Ballarin ${ }^{5}$
}

\begin{abstract}
RESUMO
O uso de florestas de Eucalyptus tem se tornado cada vez mais expressivo no Brasil, sobretudo por conta da excelente produção em termos volumétricos por elas alcançadas e pelas boas características da madeira obtida. O presente trabalho teve como objetivo identificar os melhores espaçamentos de plantio para florestas clonais de um híbrido interespecífico de Eucalyptus grandis e Eucalyptus urophylla, de forma a maximizar a produção de biomassa do fuste por hectare buscando a área vital por árvore em um intervalo que favorecesse o crescimento em diâmetro e a formação de madeira de maior densidade básica. Os dados foram disponibilizados pela empresa Duratex Florestal e são provenientes de um teste clonal exploratório de 72 meses de idade. O delineamento estatístico utilizado foi o sistemático com fator quantitativo contínuo, com 16 tratamentos e 40 repetições, considerando que a variação entre os tratamentos foi o espaçamento entre as árvores e a entrelinha possuía um espaçamento constante de três metros. Com este arranjo, o experimento possuiu área vital por árvore variando de $1,50 \mathrm{~m}^{2}$ (6.667 árvores por hectare) até $15,75 \mathrm{~m}^{2}$ (635 árvores por hectare). A maior produção em volume e biomassa do fuste por hectare ocorreu nos tratamentos com espaçamento de plantio adensado - 3 × 0,50 m; nos espaçamentos amplos, a partir de $9,00 \mathrm{~m}^{2}$ de área vital por planta, a produção de volume e biomassa do fuste por árvore foi maior. Foi observado um aumento na densidade básica ponderada da madeira até a área vital de $4,95 \mathrm{~m}^{2}$ por árvore, a partir desta área vital, a densidade básica se manteve praticamente inalterada.
\end{abstract}

Palavras-chave: espaçamento de plantio; clone de Eucalyptus; biomassa do fuste; delineamento sistemático.

\section{ABSTRACT}

The use of forests of Eucalyptus has become more expressive in Brazil, mostly because of the excellent production in volumetric terms reached by them and the good characteristics of the obtained wood. The present work was developed to search the best plantations spacings for clonal forests of a interspecific hybrid of Eucalyptus grandis and Eucalyptus urophylla, to maximize the production of the stem biomass per hectare. The data were made available by the company 'Duratex Florestal' and are proceeding from a

1 Engenheira Florestal, Doutoranda pelo Programa de Pós-Graduação em Agronomia - Energia na Agricultura, Faculdade de Ciências Agronômicas, Universidade Estadual Paulista, Rua José Barbosa de Barros, 1780, CEP 18610-307, Botucatu (SP), Brasil. mariadolores.dossantos@yahoo.com.br

2 Engenheiro Florestal, Dr., Professor do Departamento de Ciência Florestal, Faculdade de Ciências Agronômicas, Universidade Estadual Paulista, Rua José Barbosa de Barros, 1780, CEP 18610-307, Botucatu (SP), Brasil. blanco@fca.unesp.br

3 Engenheira Agronôma, Dr ${ }^{\mathrm{a}}$, Professora Voluntária do Departamento de Bioestatística, Instituto de Biociências, Universidade Estadual Paulista, Distrito de Rubião Júnior, s/n, CEP 18618-000, Botucatu (SP), Brasil. mmischan@ibb.unesp.br

4 Engenheiro Florestal, MSc., Empresa DendroTech Engenharia e Projetos Florestais, Rua Albatroz, 2222 , CEP 85015-480, Guarapuava (PR), Brasil. andre@dendrotech.com.br

5 Engenheiro Civil, Dr., Professor Titular do Departamento de Engenharia Rural, Faculdade de Ciências Agronômicas, Universidade Estadual Paulista, Rua José Barbosa de Barros, 1780, CEP 18610-307, Botucatu (SP), Brasil. awballarin@fca.unesp.br

Recebido para publicação em 17/09/2012 e aceito em 12/05/2015 
clonal test at the age of 72 months. The used statistician delineation was the systematic one with continuous quantitative factor, 16 treatments and 40 repetitions, considering that the variation between the treatments was the spacing between the trees and the space between rows was constant with three meters. Along with this arrangement, the vital area per tree was $1,50 \mathrm{~m}^{2}$ (6.667 trees per hectare) to $15,75 \mathrm{~m}^{2}$ (635 trees per hectare). The highest production volume and stem biomass per hectare occurred in spacing treatments with dense planting - 3x0,50m, in large spacings (from $9.00 \mathrm{~m}^{2}$ per plant life), it was on volume and biomass per tree stem. In this study, it was observed an increase in the weighted basic density of timber to the vital area of $4.95 \mathrm{~m}^{2}$ per tree. From this vital area, the basic density remained virtually unchanged.

Keywords: plantations spacing; Eucalyptus clone; stem biomass; systematic design.

\section{INTRODUÇÃO}

Várias empresas do setor florestal estão em busca do aumento da produção de madeira por unidade de área, tentando identificar, nos regimes de manejo, a técnica que agrega mais fortemente qualidade ao produto final por eles gerado. Entre os diversos fatores condicionantes da produção florestal, o espaçamento exerce um dos papéis fundamentais no estabelecimento, condução da floresta e custos de produção, uma vez que pode influenciar a taxa de crescimento das árvores, a qualidade da madeira, a idade de corte, bem como práticas de implantação, manejo e colheita (BALLONI; SIMÕES, 1980). O espaçamento ótimo é considerado aquele capaz de produzir o maior volume de produto em tamanho, forma e qualidade desejável (TONINI, 2003). O espaçamento tem influência marcante na produção e qualidade da madeira, sendo preciso tomar toda a precaução para decidir sobre o espaçamento inicial (BRASIL; FERREIRA, 1971).

Em florestas de ciclo curto, o espaçamento pode afetar direta e rapidamente a qualidade da madeira produzida, pois à medida que o espaçamento é reduzido, o número de árvores aumenta (maior densidade populacional) aumentando a competição entre plantas por água, luz e nutrientes devido à falta de sistema radicular e uma baixa produção fotossintética (PAULESKI, 2010). Esta competição prematura pode resultar em uma estagnação no crescimento, pois à medida que o povoamento se desenvolve, as copas se fecham e é iniciada a competição, e consequentemente, começa a manutenção ou redução da área basal (SCHNEIDER, 2008).

A prática de atividades silviculturais intensas, como o controle do espaçamento, entre outras, afetam a qualidade da madeira em decorrência do seu efeito nos padrões de crescimento das árvores (KELLISON; RUSS; FREDERICK, 1983). Dentre as práticas silviculturais adotadas, a densidade de plantio exerce grande influência tanto na produtividade quanto na qualidade da madeira, o que já foi comprovado por inúmeros estudos (COELHO; MELLO; SIMÕES, 1970; BRASIL; FERREIRA, 1971; MELLO; MASCARENHAS SOBRINHO; SIMÕES, 1971; SANTOS, 2011; SHIMOYAMA; BARRICHELO, 1989).

Nestes trabalhos, desenvolvidos entre as décadas de 70 e 80 , muito já se estudou sobre os efeitos do espaçamento de plantio em procedências de Pinus e Eucalyptus. Uma das grandes dificuldades encontradas pelos autores era que as interações estatisticamente significativas ocorriam ao nível de genótipo, e não a diferentes espaçamentos, conforme comentado por Ferreira (1968) e Mello, Mascarenhas Sobrinho e Simões (1976). Mesmo em trabalhos em que se buscava detectar a ocorrência de interações competitivas entre procedências nos diferentes espaçamentos, os autores encontravam interações entre o espaçamento e o grau de variação genética (BALLONI, 1978, JACOB; BALLONI, 1978), mas para o caso de interação genótipo-espaçamento, não era detectado nenhum grau de significância.

Atualmente, com a introdução das florestas clonais, novos estudos têm sido realizados na tentativa de esclarecer o comportamento do crescimento destas florestas que apresentam um desenvolvimento em diâmetro e altura mais homogêneo. Thiersch (2002), estudando a relação da densidade básica em função do diâmetro, observou resultados opostos para os clones de Eucalyptus sp., identificando que a densidade básica da madeira aumentou no sentido dos menores para os maiores diâmetros para o clone 1 e comportamento contrário foi observado no clone 2 no índice de sítio mais produtivo, havendo um decréscimo desta variável com o aumento do diâmetro a 1,30 m do solo. Goulart et al. (2003) estudaram as variáveis densidade básica, massa seca da madeira e de casca para Eucalyptus grandis em função de 12 espaçamentos no Rio Grande do Sul e observaram que a maior produção de massa seca de casca e de madeira por unidade de área estava relacionada com o número de árvores por hectare e também com a densidade básica da madeira. 
O presente trabalho foi desenvolvido buscando a identificação dos melhores espaçamentos de plantio para florestas clonais de um híbrido interespecífico de Eucalyptus grandis e Eucalyptus urophylla, de forma a maximizar a produção de biomassa do fuste por hectare.

\section{MATERIAL E MÉTODO}

\section{Delineamento experimental}

O estudo foi realizado em uma unidade florestal pertencente à empresa Duratex Florestal, localizada no município de Lençóis Paulista - SP, com as coordenadas geográficas SAD69 e MC $51^{\circ} \mathrm{Wgr}$

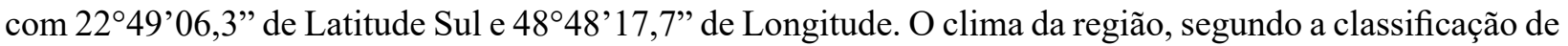
Köppen, é do tipo CWA com médias anuais próximas a $1.330 \mathrm{~mm}$ de precipitação e $21^{\circ} \mathrm{C}$ de temperatura. O solo é classificado como Latossolo Vermelho Distrófico Psamítico, textura média arenosa, A moderado, Álico, Caulinítico. Os dados analisados são provenientes de uma área experimental que fazia parte de uma série de ensaios de espaçamento instalados em diferentes regiões de atuação da empresa, que receberam o manejo silvicultural usual dos plantios comerciais da empresa.

O delineamento estatístico utilizado é denominado delineamento sistemático com fator quantitativo contínuo. Consiste no plantio das árvores em linhas com espaçamento constante e proporcionando um aumento gradual no espaçamento entre as árvores. Delineamento semelhante já fora proposto por Nelder (1962), denominado delineamento sistemático tipo leque, com o plantio das árvores em raios, aumentandose a partir do centro do experimento o espaçamento entre linhas e entre árvores.

Neste estudo, o espaçamento adotado entre linhas foi de 3,0 $\mathrm{m}$ e o espaçamento entre árvores variou de $0,50 \mathrm{~m}$ a 5,25 m. Utilizou-se um clone híbrido interespecífico de Eucalyptus grandis e Eucalyptus urophylla, configurando-se 16 tratamentos com 40 árvores por tratamento, podendo-se associar a cada espaçamento uma área vital que variou de $1,50 \mathrm{~m}^{2} /$ árvore (6.667 árvores por hectare) até $15,75 \mathrm{~m}^{2} /$ árvore (635 árvores por hectare), conforme apresentado na Tabela 1.

TABELA 1: Tratamentos utilizados no experimento.

TABLE 1: The utilized treatments in the experiment.

\begin{tabular}{cccc}
\hline Tratamento & $\begin{array}{c}\text { Espaçamento }(\mathrm{m}) \\
\text { (entre linha } \text { × entre árvore) }\end{array}$ & Área vital $\left(\mathrm{m}^{2}\right)$ & Lotação (árvores/ha) \\
\hline 1 & $3 \times 0,50$ & 1,50 & 6.667 \\
2 & $3 \times 0,55$ & 1,65 & 6.061 \\
3 & $3 \times 0,65$ & 1,95 & 5.128 \\
4 & $3 \times 0,75$ & 2,25 & 4.444 \\
5 & $3 \times 0,90$ & 2,70 & 3.704 \\
6 & $3 \times 1,10$ & 3,30 & 3.030 \\
7 & $3 \times 1,35$ & 4,05 & 2.469 \\
8 & $3 \times 1,65$ & 4,95 & 2.020 \\
9 & $3 \times 1,95$ & 5,85 & 1.709 \\
10 & $3 \times 2,25$ & 6,75 & 1.481 \\
11 & $3 \times 2,60$ & 7,80 & 1.282 \\
12 & $3 \times 3,00$ & 9,00 & 1.111 \\
13 & $3 \times 3,40$ & 10,20 & 980 \\
14 & $3 \times 3,80$ & 11,40 & 877 \\
15 & $3 \times 4,25$ & 12,75 & 784 \\
16 & $3 \times 5,25$ & 15,75 & 635 \\
\hline
\end{tabular}




\section{Coleta dos dados}

\section{Volume, densidade básica da madeira e biomassa do fuste}

As 40 árvores de cada um dos tratamentos foram acompanhadas ao longo dos anos em termos de medições anuais de diâmetro e altura. Aos 72 meses de idade, idade de corte do povoamento, três árvores que apresentassem parâmetros representativos da média do tratamento foram amostradas e derrubadas para determinação dos volumes individuais (e estimativa do volume por hectare) e da densidade básica ponderada da árvore. A biomassa do fuste, considerada até o diâmetro mínimo de quatro centímetros, foi determinada multiplicando-se o valor do volume pela densidade básica ponderada da madeira, em cada tratamento.

As árvores selecionadas foram derrubadas e as medidas de diâmetro foram tomadas de metro em metro, da base de corte até o diâmetro mínimo de quatro centímetros, calculando-se o volume de cada seção pela fórmula proposta por Smalian. Para estimativa do volume total por hectare, o volume médio das três árvores representativas de cada tratamento foi extrapolado pelo número de árvores por hectare (lotação/ha) para os tratamentos avaliados.

A determinação da densidade básica ponderada das árvores amostradas seguiu metodologia proposta por Rezende (1997). Foram retirados três discos de $5 \mathrm{~cm}$ a $12,5 \%$ (disco A), 37,5\% (disco B) e 62,5\% (disco C) da altura total da árvore e, a partir deles, determinaram-se as respectivas densidades básicas pelo método de deslocamento de água.

O volume do fuste da árvore (v) foi obtido pelo somatório dos volumes das " $n$ " seções ao longo da altura total da árvore acrescido do volume da ponta (Equação 1)

$$
v=\left[\left(\frac{g_{1}+g_{2}}{2}\right) \ell\right]+\left[\left(\frac{g_{2}+g_{3}}{2}\right) \ell\right]+\ldots+\left[\left(\frac{g_{n-1}+g_{n}}{2}\right) \ell\right]+v_{p} \quad v_{p}=\frac{g_{n} \ell_{p}}{3}
$$

Em que: $v=$ volume (1) do fuste da árvore; $g_{1}=$ área transversal na base da seção; $g_{2}=$ área transversal no topo da seção; $\ell=$ comprimento da seção; $v_{p}=$ volume da ponta Equação 2.

A densidade básica ponderada da madeira de cada árvore $\left(D b_{\mathrm{POND}}\right)$ foi calculada utilizando-se a

$$
D b_{\text {POND }}=\frac{A_{A} D b_{A}+A_{B} D b_{B}+A_{C} D b_{C}}{A_{A}+A_{B}+A_{C}}
$$

Em que: $D b_{\text {POND }}=$ densidade básica ponderada da(2)adeira da árvore; $\mathrm{A}=$ área transversal dos discos (A, $\mathrm{B}$ e C); $D b=$ densidade dos discos (A, B e C).

\section{Análise dos dados}

Os dados obtidos foram previamente analisados pelo Teste de Levene (LEVENE, 1960) para verificação da homogeneidade das variâncias dos tratamentos.

Em função da ausência de casualização dos delineamentos sistemáticos e de não se deixar bordaduras entre os tratamentos, eles não podem ser analisados como se fossem delineamentos aleatórios (MEAD, 1988 apud STAPE, 1995). Evert (1971), Lin e Morse (1975) comentam que quase inexistem trabalhos efetuando análises estatísticas de delineamentos sistemáticos. Segundo Stape (1995), diversos autores recomendam a análise de regressão como uma das grandes ferramentas de análise de delineamentos sistemáticos com níveis crescentes do fator nos tratamentos. Silva. Meunier e Ferraz (1996) afirmam que, atualmente, um dos modelos mais utilizados em pesquisas que avaliam o crescimento de florestas é o modelo de Chapman-Richards. Venus e Causton (1979) recomendam o uso deste modelo pela flexibilidade e por fornecer parâmetros com significado biológico. Na Tabela 2 são apresentados os modelos adotados 
TABELA 2: Modelos de resposta ao espaçamento, adotados para as variáveis com correlação positiva com o espaçamento: volume individual das árvores, densidade básica da madeira e biomassa do fuste da árvore.

TABLE 2: Response models assumed for the variables with positive correlation to planting spacing: individual volume, basic density and stem biomass.

\begin{tabular}{lll}
\hline \multicolumn{1}{c}{ Modelo } & \multicolumn{1}{c}{ Função } & Equação \\
\hline Von Bertalanffy & $y=\beta_{0}\left(1-\beta_{1}^{x}\right)^{3}$
\end{tabular}

Em que: $y=$ volume do fuste por árvore, densidade básica da madeira ou biomassa do fuste por árvore; $x=$ área vital por tratamento; $\beta_{i}=$ parâmetros da equação.

para a análise dos dados de volume por árvore, densidade básica da madeira e biomassa do fuste da árvore, variáveis que apresentaram correlação positiva com o espaçamento.

$\mathrm{Na}$ análise dos dados que apresentaram correlação negativa com o espaçamento - volume por hectare e biomassa do fuste por hectare - foram utilizadas as funções exponencial e hiperbólica (Tabela 3), que são frequentemente utilizadas para ajustar dados de experimentos biológicos.

Estas funções são preferencialmente utilizadas ao invés de modelos polinomiais pelo fato de que o limite da função exponencial, quando o espaçamento $(x)$ cresce indefinidamente, é zero se $\beta_{l}<1$ e infinito se $\beta_{l}>1$. Isto porque a derivada não se anula para nenhum valor de $x \mathrm{e}$, portanto, a função não possui pontos extremos (máximos ou mínimos), como é o caso dos polinômios de grau maior ou igual a 2 . A função hiperbólica é indicada ainda quando se espera que os valores da variável dependente $y$ aproximemse assintoticamente de zero quando $x$ tende a infinito; esta tendência, segundo Mischan e Pinho (1996) pode ser uma vantagem sobre os modelos polinomiais, uma vez que biologicamente não se espera, em muitos

TABELA 3: Modelos de resposta ao espaçamento adotados para as variáveis com correlação negativa com o espaçamento: volume por hectare e biomassa do fuste por hectare.

TABLE 3: Response models assumed for variables with negative correlation to planting spacing: volume per hectare and stem biomass per hectare.

\begin{tabular}{lll}
\hline \multicolumn{1}{c}{ Modelo } & Função & Equação \\
\hline Exponencial & $y=\beta_{0} e^{\beta_{1} x}$ & (9) \\
\hline Hiperbólico & $y=\frac{\beta_{0}}{x^{\beta_{1}}}$
\end{tabular}

Em que: $y=$ volume do fuste por hectare ou biomassa do fuste por hectare; $x=$ espaçamento por tratamento; $\beta_{i}=$ parâmetros da equação. 
casos, que haja pontos extremos.

A seleção do melhor modelo para cada variável dependente analisada foi baseada no maior coeficiente de determinação ajustado $\left(R_{\text {Ajust. }}^{2}\right)$, na estimativa do erro padrão dos resíduos absolutos $(\operatorname{Syx})$ e no erro padrão dos resíduos relativos (Syx \%) (SCOLFORO, 2005). Embora não seja considerada uma medida de precisão na seleção dos modelos, Scolforo (2005) e Campos e Leite (2006) recomendam a análise de distribuição gráfica dos valores residuais (Res\%). Segundo os autores é critério importante e fundamental para a seleção do modelo de regressão ajustado e decisivo na avaliação da qualidade das estimativas por permitir detectar se há ou não tendência na estimativa da variável dependente ao longo de toda linha de regressão.

\section{RESULTADOS E DISCUSSÃO}

\section{Volume do fuste por árvore e por hectare}

Para a variável volume do fuste por árvore, os modelos testados apresentaram coeficientes de determinação e erro padrão dos resíduos muito próximos (Tabela 4). Assim, a seleção do melhor modelo foi com base na distribuição gráfica dos resíduos. O modelo de Chapman-Richards foi o que apresentou a menor variação dos resíduos com relação à média, com desempenho ligeiramente superior aos demais para o volume do fuste por árvore.

TABELA 4: Coeficientes e estatísticas das equações ajustadas para o volume do fuste por árvore em função da área vital.

TABLE 4: Coefficients and statistics of the adjusted equations for the tree stem volume as a function of tree vital area.

\begin{tabular}{|c|c|c|c|c|c|c|c|}
\hline \multirow{2}{*}{ Modelo } & \multirow{2}{*}{ Equação } & \multicolumn{3}{|c|}{ Coeficientes } & \multirow{2}{*}{$R_{\text {Ajust. }}^{2}(\%)$} & \multirow{2}{*}{$S_{y x}$} & \multirow{2}{*}{$S_{y x}(\%)$} \\
\hline & & $\beta_{0}$ & $\beta_{1}$ & $\beta_{2}$ & & & \\
\hline Von Bertalanffy & 3 & 0,8877 & 0,0172 & & 95,26 & 0,0307 & 12,97 \\
\hline Chapman-Richards & 4 & 0,8877 & 0,4129 & 0,1253 & 95,71 & 0,0284 & 12,11 \\
\hline Weibull & 5 & 0,5899 & 2,5520 & 0,1658 & 95,34 & 0,0296 & 12,63 \\
\hline Gompertz & 6 & 0,5234 & 7,5208 & 0,2863 & 94,87 & 0,0311 & 13,27 \\
\hline Brody & 7 & 1,2029 & 0,0413 & 0,9289 & 95,82 & 0,0279 & 11,89 \\
\hline Logística & 8 & 0,9451 & 0,0052 & 0,0470 & 95,81 & 0,0277 & 11,83 \\
\hline
\end{tabular}

Em que: $\beta_{i}=$ parâmetros da equação, $R_{A j u s t .}^{2}(\%)=$ coeficiente de determinação ajustado, $S y x=$ erro padrão dos resíduos absolutos e Syx $(\%)=$ erro padrão dos resíduos relativos.

Analisando o modelo de Chapman-Richards ajustado (Figura 1), observa-se que o volume por árvore foi superior nos espaçamentos mais amplos, ou seja, aqueles que proporcionaram maior espaço vital às árvores $\left(15,75 \mathrm{~m}^{2}\right)$ apresentaram indivíduos com maior volume $\left(0,5178 \mathrm{~m}^{3}\right)$. Este resultado está de acordo com Pinkard e Neilsen (2003) que encontraram maior volume por árvore devido ao maior DAP em povoamento com 500 árvores de Eucalyptus nitens por hectare, comparado às densidades populacionais de até 1.667 árvores por hectare. Resultado semelhante também foi obtido por Oliveira-Neto et al. (2003) trabalhando com Eucalyptus camaldulensis com 32 meses de idade, cujos maiores valores de área vital proporcionam maior produção de madeira por árvore devido à menor competição entre os indivíduos no povoamento. Na idade da avaliação - 72 meses - observa-se que a regressão ajustada não apresenta estabilização até a área vital de $15,75 \mathrm{~m}^{2}$ (espaçamento 3 x 5,25 m), sugerindo uma resposta positiva do clone com relação à produção individual volumétrica também para espaçamentos superiores aos estudados.

Neste estudo, a maior produção volumétrica por árvore correspondeu também ao maior desenvolvimento em diâmetro e altura das árvores nos maiores espaçamentos, resultados que estão em concordância com Schönau (1974), Fishwick (1976), Couto, Brandi e Condé (1977), Berger (2000), entre 


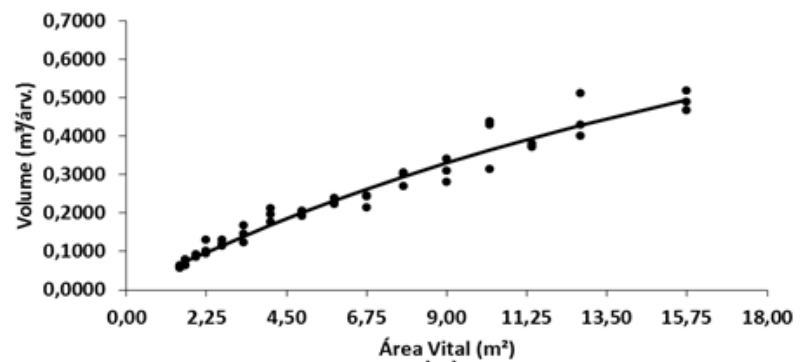

(a)

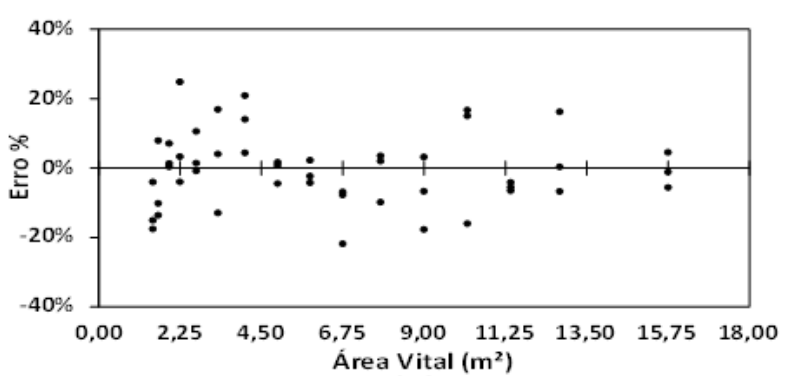

(b)

FIGURA 1: (a) Volume por árvore (aos 72 meses) em função da área vital dos tratamentos e representação gráfica do modelo de Chapman-Richards ajustado; (b) Distribuição dos resíduos para volume por árvore (aos 72 meses) em função do espaçamento ajustado com o modelo de ChapmanRichards.

FIGURE 1: (a) Tree volume (in 72 months) per vital area of the treatments and graphical representation of the Chapman-Richards model adjusted; (b) Distribution of the residues for the volume per tree (age of 72 months) based on the spacing adjusted with the model of Chapman-Richards.

outros.

Pela Tabela 5 pode-se observar no volume por hectare um decréscimo com o aumento do espaçamento. Em uma análise inicial, baseada exclusivamente nos parâmetros estatísticos das regressões, evidencia-se a superioridade do modelo exponencial (Figura 2-a); entretanto, com o auxilio da Figura 2-c, pode-se observar que o modelo que representa o comportamento biológico natural para uma floresta é o hiperbólico. Isto porque, como a produção em volume por hectare não tende a zero com o aumento do espaçamento, a regressão ajustada com o modelo exponencial torna-se frágil. Nesta situação, Mischan e Pinho (1996) afirmam que a função hiperbólica vem sendo empregada para ajustar os dados de experimentos nos quais não se esperam os pontos extremos, mas sim um comportamento assintótico quando o espaçamento aumenta consideravelmente.

A maior produtividade por hectare ocorreu nos espaçamentos adensados - Tratamentos 1 a 6 (áreas vitais de $1,50 \mathrm{~m}^{2}$ a $\left.3,30 \mathrm{~m}^{2}\right)$, logo o espaçamento $3 \times 0,50 \mathrm{~m}$ que possuía a maior lotação de árvores por hectare (6.667 árv./ha) obteve maior produção volumétrica; e a menor produção ocorreu no espaçamento 3 x 5,25 m. Para o volume por hectare, os valores variaram de $290,29 \mathrm{~m}^{3} / \mathrm{ha}$ a $479,87 \mathrm{~m}^{3} /$ ha, nos espaçamentos $3 \times 5,25 \mathrm{~m}$ e $3 \times 0,50 \mathrm{~m}$, respectivamente. Este resultado concorda com os estudos de Vital e Della Lucia (1987) e Garcia, Corradine e Alvarenga (1991), que encontraram influência significativa do espaçamento sobre a produção em volume por hectare.

Couto, Brandi e Condé (1977) observaram que o volume médio produzido por hectare para Eucalyptus urophylla foi influenciado pelo espaçamento, em todas as idades estudadas, sendo que as

TABELA 5: Coeficientes e estatísticas das duas equações ajustadas para o volume por hectare das 48 árvores medidas aos 72 meses.

TABLE 5: Coefficients and statistics of the two equations adjusted for the volume per hectare of the 48 trees measured at 72 months.

\begin{tabular}{|c|c|c|c|c|c|c|}
\hline \multirow{2}{*}{ Modelo } & \multirow{2}{*}{ Equação } & \multicolumn{2}{|c|}{ Coeficientes } & \multirow{2}{*}{$R_{\text {Ajust. }}^{2}(\%)$} & \multirow{2}{*}{$S_{y x}$} & \multirow{2}{*}{$S_{y x}(\%)$} \\
\hline & & $\beta_{0}$ & $\beta_{1}$ & & & \\
\hline Exponencial & 9 & 469,3577 & 0,9690 & 78,52 & 26,7701 & 6,91 \\
\hline Hiperbólico & 10 & 496,0347 & 0,1587 & 70,20 & 31,5401 & 8,14 \\
\hline
\end{tabular}




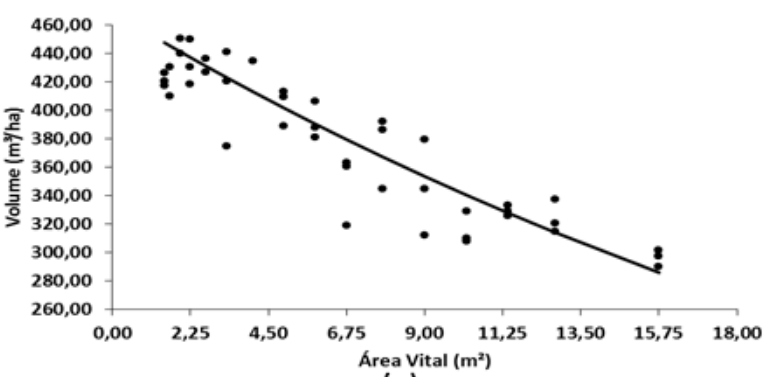

(a)

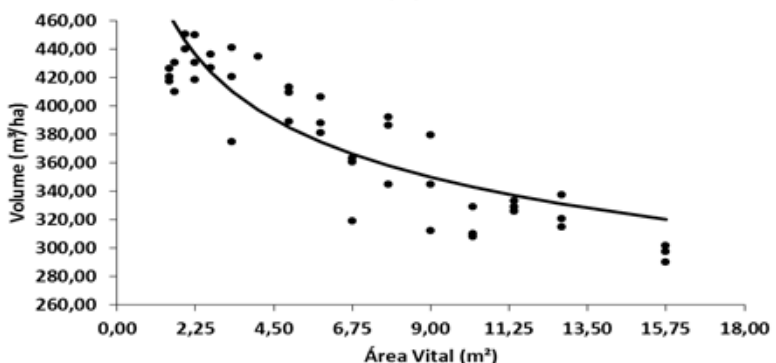

Areavitis
(c)

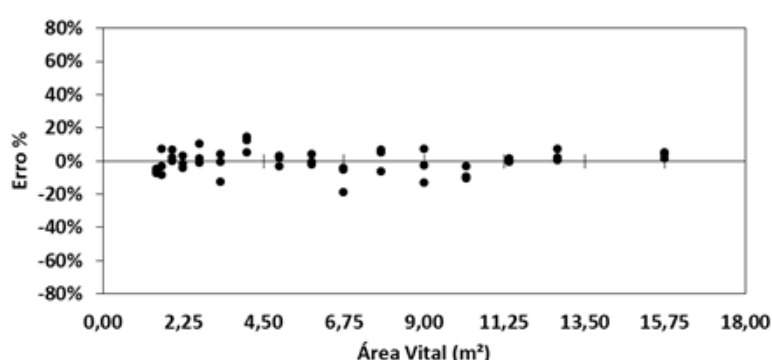

(b)

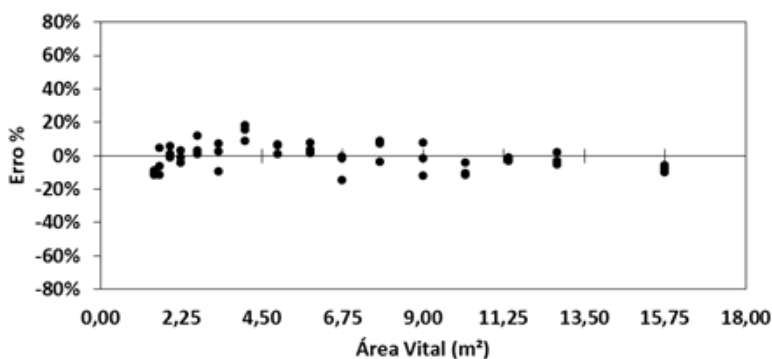

(d)

FIGURA 2: (a) Volume por hectare (aos 72 meses) em função da área vital dos tratamentos e representação gráfica do modelo exponencial ajustado; (b) Distribuição dos resíduos para volume por hectare (aos 72 meses) em função do espaçamento ajustado com o modelo exponencial; (c) Volume por hectare (aos 72 meses) em função da área vital dos tratamentos e representação gráfica do modelo hiperbólico ajustado; (d) Distribuição dos resíduos para volume por hectare (aos 72 meses) em função do espaçamento ajustado com o modelo hiperbólico.

FIGURE 2: (a) Volume per hectare (age of 72 months) per vital area of the treatments and graphical representation of the exponential model adjusted; (b) Distribution of the residues for the volume per hectare (age of 72 months) based on the spacing adjusted with the exponential model; (c) Volume per hectare (age of 72 months) per vital area of the treatments and graphical representation of the hyperbolic model adjusted; (d) Distribution of the residues for the volume per hectare (72 months) based on the spacing adjusted with the hyperbolic model.

menores produções foram encontradas nos povoamentos com menor densidade de plantio. Esta tendência de diminuir a produção em volume por hectare com o aumento do espaçamento, também foi observada por Botelho (1998) e Oliveira-Neto et al. (2003), em que a maior produção por unidade de área ocorre nos espaçamentos mais adensados em função do maior número de indivíduos.

\section{Densidade básica média ponderada das árvores}

Os modelos ajustados para a variável densidade básica média ponderada das árvores apresentaram coeficientes de determinação ajustado e erro padrão dos resíduos muito próximos (Tabela 6). Assim, novamente aqui, fez-se uso do critério proposto por Scolforo (2005) e Campos e Leite (2006), sendo selecionado o modelo de Von Bertalanffy, que além de possuir o maior coeficiente de determinação ajustado, foi o que apresentou a menor variação dos resíduos com relação à média.

Aos 72 meses de idade foi observada uma variação entre tratamentos na densidade básica média ponderada das árvores de $441,68 \mathrm{~kg} / \mathrm{m}^{3}$ a $492,56 \mathrm{~kg} / \mathrm{m}^{3}$ para os espaçamentos $3 \times 0,50 \mathrm{~m}$ e $3 \times 5,25$ $\mathrm{m}$, respectivamente. Observa-se que os espaçamentos mais adensados, aqui considerados de 1 a 6 , são caracterizados por árvores que apresentaram menores volumes (Figura 1), bem como as menores densidades básicas médias ponderadas na idade da avaliação, sofrendo aumento na densidade básica com o aumento da área vital por árvore (Figura 3). O aumento na densidade básica foi observado até a área vital de 4,95 $\mathrm{m}^{2}$ /árvore (espaçamento $3 \times 1,65 \mathrm{~m}$ ), a partir dessa área vital, a densidade básica se manteve praticamente inalterada até os espaçamentos mais amplos. 
Este comportamento bem definido em duas regiões distintas (espaçamentos mais e menos adensados) pode ser atribuído ao material genético homogêneo que foi estudado, diferente do obtido em estudos de plantios seminais.

Os resultados apresentados neste estudo estão em desacordo com os obtidos por Garcia, Corradine e Alvarenga (1991) estudando plantios seminais de Eucalyptus saligna e Eucalyptus grandis em que os mesmos concluíram que a densidade básica tende a diminuir com o aumento do espaçamento. Ferreira (1968) e Souza, Della Lucia e Resende (1979) também comentam o aumento da densidade básica em árvores mais vigorosas, característica dos maiores espaçamentos. $\mathrm{O}$ mesmo fato foi observado por Ferreira e Kageyama (1978), que concluíram que as árvores mais vigorosas de populações híbridas de Eucalyptus grandis e Eucalyptus saligna apresentavam maior densidade. Mais recentemente, Berger (2000) observou um aumento gradual na densidade com o aumento do espaço vital em plantios clonais de Eucalyptus saligna (área vital de 6 a $12 \mathrm{~m}^{2}$ ).

\section{Biomassa do fuste por árvore e por hectare}

Para a variável dependente biomassa do fuste por árvore, os modelos testados novamente apresentaram coeficientes de determinação e erro padrão residual muito próximos (Tabela 7); assim, também nesse caso, a seleção do melhor modelo foi feita com base na distribuição gráfica dos resíduos. O modelo de Chapman-Richards ajustado, apesar de não apresentar o maior $R_{\text {Ajust. }}^{2}$, foi o que apresentou a menor variação na distribuição dos resíduos com relação a média.

A Figura 4, que retrata a variação da biomassa do fuste por árvore com o aumento do espaçamento de plantio, permite concluir que os espaçamentos mais amplos foram os que proporcionaram maior produção de biomassa do fuste por árvore, pois o aumento da área vital por planta reduziu a lotação por unidade de área e proporcionou o desenvolvimento de árvores de maiores dimensões, tanto em diâmetro quanto em altura.

Diante dos dados coletados para este trabalho, foi possível observar um aumento na altura média das árvores à medida que o espaçamento era ampliado. Foi possível observar ainda a uniformização das alturas ocorrendo mais rapidamente nos tratamentos com espaçamento amplo, que corresponderam aos Tratamentos 12 ao 16, atingindo seu valor assintótico em um espaçamento de aproximadamente $9,0 \mathrm{~m}^{2}$ de área vital por árvore. Entende-se que em uma floresta clonal, objeto de análise deste estudo, instalada em solo homogêneo de igual capacidade produtiva de sítio; quanto maior a área disponível para o crescimento de uma árvore, maior será a disponibilidade de nutrientes e água, o que contribui para o seu maior desenvolvimento individual; o contrário ocorre em uma floresta adensada, onde a concorrência por nutrientes e água é mais acentuada em função da proximidade das árvores, aumentando a competição entre elas. As maiores alturas obtidas nos espaçamentos amplos contrariam as constatações de Patino-Valera

TABELA 6: Coeficientes e estatísticas das equações ajustadas para densidade básica ponderada das árvores. TABLE 6: Coefficients and statistics of the equations adjusted for the balanced specific gravity of the 48 trees measured at 72 months.

\begin{tabular}{|c|c|c|c|c|c|c|c|}
\hline \multirow{2}{*}{ Modelo } & \multirow{2}{*}{ Equação } & \multicolumn{3}{|c|}{ Coeficientes } & \multirow{2}{*}{$R_{\text {Ajust. }}^{2}(\%)$} & \multirow{2}{*}{$S_{y x}$} & \multirow{2}{*}{$S_{y x}(\%)$} \\
\hline & & $\beta_{0}$ & $\beta_{1}$ & $\beta_{2}$ & & & \\
\hline Von Bertalanffy & 3 & 486,7347 & 0,5127 & & 55,61 & 13,3576 & 2,79 \\
\hline Chapman-Richards & 4 & 486,7347 & 0,1653 & 9,3003 & 54,60 & 13,5085 & 2,83 \\
\hline Weibull & 5 & 486,0006 & 1,3358 & 1,2838 & 55,22 & 13,4136 & 2,81 \\
\hline Gompertz & 6 & 486,1586 & 2,2593 & 2,0086 & 55,18 & 13,4206 & 2,81 \\
\hline Logística & 8 & 486,1226 & 2,7087 & 2,09178 & 55,25 & 13,4098 & 2,80 \\
\hline
\end{tabular}




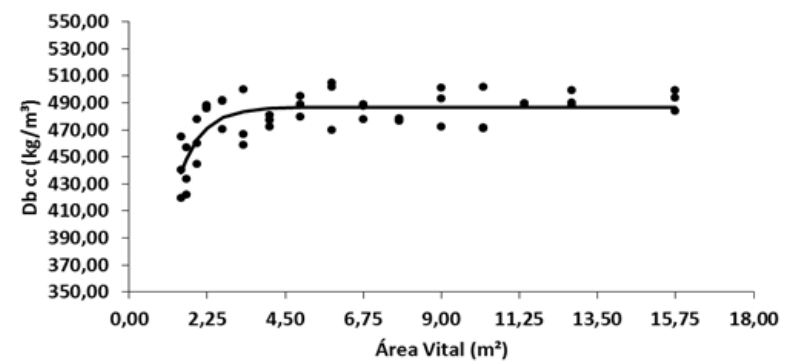

(a)

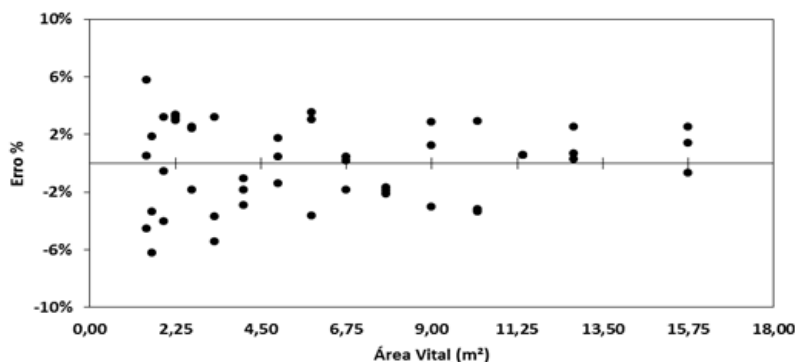

(b)

FIGURA 3: (a) Densidade básica da madeira com casca (Db cc) aos 72 meses em função da área vital dos tratamentos e representação gráfica do modelo de Von Bertalanffy ajustado; (b) Distribuição dos resíduos para volume por árvore em função do espaçamento ajustado com o modelo de Von Bertalanffy, aos 72 meses.

FIGURE 3: (a) Basic density in 72 months per vital area of the treatments and graphical representation of the Von Bertalanffy model adjusted; (b) Distribution of the residues for the basic density based on the spacing adjusted with the model of Von Bertalanffy, at 72 months.

(1986), Bernardo (1995) e Assis et al. (1999), pois, segundo estes autores, o maior crescimento inicial em altura das árvores ocorre em espaçamentos menores. Porém, Balloni e Simões (1980) afirmam que existem casos em que a altura média aumenta com o espaçamento e outros em que o resultado é inverso. Coelho, Mello e Simões (1970) constataram que os espaçamentos tiveram efeito positivo sobre o crescimento em diâmetro, não influenciando o crescimento em altura das árvores.

Para a produção de biomassa do fuste por árvore foi observada uma grande variação entre os tratamentos extremos. O Tratamento $1 \mathrm{com} 1,50 \mathrm{~m}^{2}$ de área vital possuía árvores de menores dimensões e com biomassa do fuste das árvores em torno de 29,64 kg/árvore, enquanto o Tratamento 16 , com 15,75 m² de área vital, possuía árvores de maiores dimensões e com biomassa do fuste das árvores em torno de 242 $\mathrm{kg} /$ árvore, uma variação de $212 \mathrm{~kg} /$ árvore na biomassa do fuste entre os tratamentos extremos. A maior produção de biomassa do fuste por árvore também apresentou resposta linear positiva para a variável área útil por árvore nos estudos de Oliveira-Neto et al. (2003), trabalhando com Eucalyptus camaldulensis aos 32 meses de idade. Segundo os autores, ainda neste estudo, a área que proporcionou maior produção de madeira por árvore foi de $15 \mathrm{~m}^{2}$.

TABELA 7: Coeficientes e estatísticas das seis equações ajustadas para a biomassa do fuste por árvore para as 48 árvores amostradas aos 72 meses.

TABLE 7: Coefficients and statistics of the six equations adjusted for the stem biomass of the 48 trees measured at 72 months.

\begin{tabular}{|c|c|c|c|c|c|c|c|}
\hline \multirow{2}{*}{ Modelo } & \multirow{2}{*}{ Equação } & \multicolumn{3}{|c|}{ Coeficientes } & \multirow{2}{*}{$R^{2}{ }_{\text {Ajust. }}(\%)$} & \multirow{2}{*}{$S_{y x}$} & \multirow{2}{*}{$S_{y x}(\%)$} \\
\hline & & $\beta_{0}$ & $\beta_{1}$ & $\beta_{2}$ & & & \\
\hline Von Bertalanffy & 3 & 453,5371 & 0,0163 & & 96,56 & 12,4353 & 10,86 \\
\hline Chapman-Richards & 4 & 453,5371 & 0,1847 & 0,2642 & 96,48 & 12,5758 & 10,98 \\
\hline Weibull & 5 & 685,2875 & 0,0352 & 0,9197 & 96,52 & 12,4795 & 10,90 \\
\hline Gompertz & 6 & 296,5057 & 2,5550 & 0,1597 & 96,17 & 13,1001 & 11,44 \\
\hline Brody & 7 & 514,0564 & 0,0082 & 0,0406 & 96,59 & 12,2339 & 10,68 \\
\hline Logística & 8 & 260,4589 & 7,5657 & 0,2797 & 95,72 & 13,8712 & 12,11 \\
\hline
\end{tabular}

Em que: $\beta_{i}=$ parâmetros da equação, $R_{A j u s t .}^{2}(\%)=$ coeficiente de determinação ajustado, $S y x=$ erro padrão dos resíduos absolutos e Syx $(\%)=$ erro padrão dos resíduos relativos. 


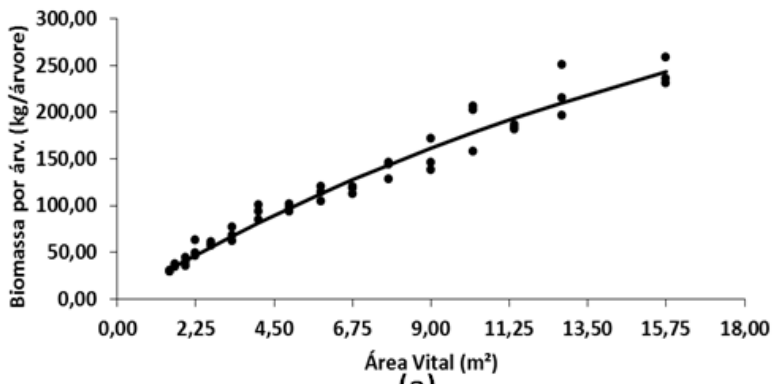

(a)

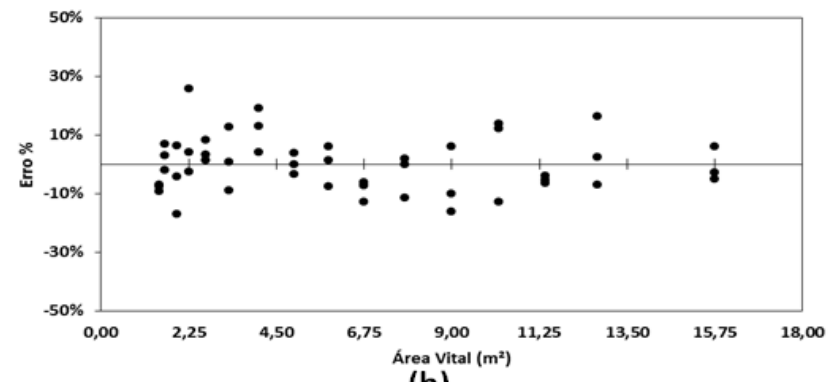

(b)

FIGURA 4: (a) Biomassa do fuste por árvore aos 72 meses em função da área vital dos tratamentos e representação gráfica do modelo de Chapman-Richards ajustado; (b) Distribuição dos resíduos para volume por árvore em função do espaçamento ajustado com o modelo de ChapmanRichards, aos 72 meses.

FIGURE 4: (a) Stem biomass per tree in 72 months per vital area of the treatments and graphical representation of the Chapman-Richards model adjusted; (b) Distribution of the residues for the stem biomass per tree based on the spacing adjusted with the model of Chapman-Richards, at 72 months.

A Tabela 8 e a Figura 5 apresentam o comportamento da biomassa do fuste por hectare com o aumento da área vital por planta. Em uma análise prévia, baseada apenas nos parâmetros estatísticos da análise de regressão, evidencia-se que o modelo exponencial foi superior ao modelo hiperbólico; entretanto, pode-se observar que o modelo exponencial é incapaz de captar a curvatura natural da relação biomassaespaçamento de uma floresta, por não ser biologicamente fundamentado para a variável biomassa do fuste por hectare. Assim, novamente optou-se pelo uso do modelo hiperbólico (MISCHAN; PINHO, 1996) que melhor representou a biomassa do fuste por hectare por se tratar de uma variável que possui um comportamento assintótico com o aumento do espaçamento, não tendendo a zero.

Na Figura 5-c é apresentado o modelo hiperbólico ajustado para a produção de biomassa do fuste por hectare e, com base nos dados, evidenciou-se que nos espaçamentos adensados, em que se tem um maior número de árvores por hectare, houve também maior produção de biomassa do fuste por hectare. A variação na produção de biomassa do fuste por hectare entre os tratamentos extremos - 1 e 16 - foi de 81 ton/ ha, sendo que o Tratamento $1 \mathrm{com} 1,50 \mathrm{~m}^{2}$ de área vital a produção média foi 225 ton de biomassa do fuste por hectare e no Tratamento $16 \mathrm{com} 15,75 \mathrm{~m}^{2}$ de área vital a produção foi em torno de 153 ton de biomassa do fuste por hectare.

A partir da análise da Figura 5-c identifica-se que a maior produção em biomassa do fuste por hectare de forma que não haja perdas de crescimento em diâmetro na floresta, as áreas vitais mais adequadas variaram de $3,30 \mathrm{~m}^{2}$ a 4,95 $\mathrm{m}^{2}$ (espaçamentos de plantio de $3 \times 1,10 \mathrm{~m}$ e de 3 x 1,65 m, respectivamente); da mesma forma, os ganhos em densidade básica da madeira sem perdas expressivas na produção de biomassa

TABELA 8: Coeficientes e estatísticas das duas equações ajustadas para a biomassa do fuste por hectare para as 48 árvores amostradas aos 72 meses.

TABLE 8: Coefficients and statistics of the two equations adjusted for the stem biomass per hectare of the 48 trees measured at 72 months.

\begin{tabular}{ccccccc}
\hline \multirow{2}{*}{ Modelo } & \multirow{2}{*}{ Equação } & \multicolumn{2}{c}{ Coeficientes } & \multirow{2}{*}{$R^{2}{ }_{A j u s t .}(\%)$} & $S_{y x}$ & \multirow{2}{*}{$S_{y x}(\%)$} \\
\cline { 2 - 5 } & & $\beta_{0}$ & $\beta_{1}$ & & & \\
\hline Exponencial & 9 & 227,7359 & 0,9725 & 74,70 & 12,7474 & 6,64 \\
Hiperbólico & 10 & 240,7111 & 0,1451 & 69,45 & 14,0109 & 7,30 \\
\hline
\end{tabular}

Em que: $\beta_{i}=$ parâmetros da equação, $\left.R_{A j u s t .}^{2} \%\right)=$ coeficiente de determinação ajustado, Syx = erro padrão dos resíduos absolutos e Syx (\%) = erro padrão dos resíduos relativos. 


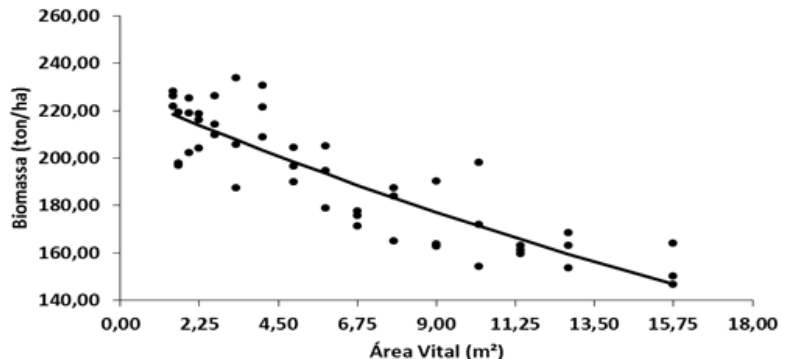

(a)

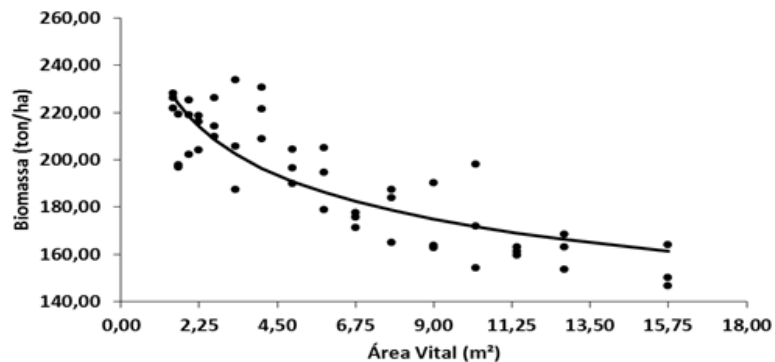

(c)

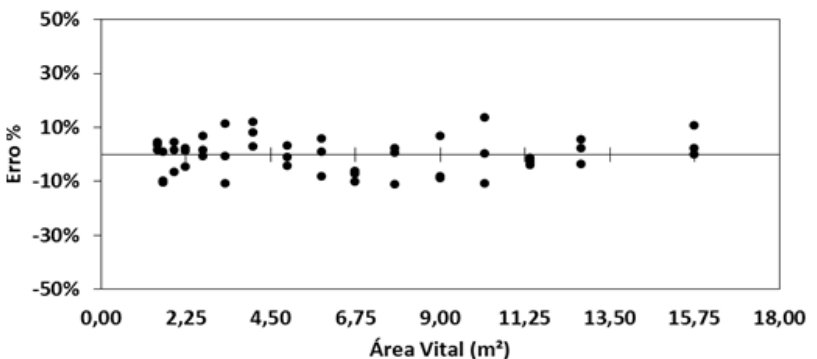

(b)

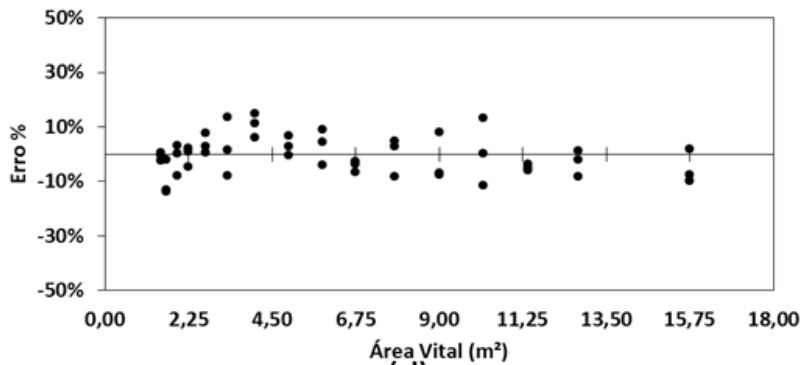

(d)

FIGURA 5: (a) Biomassa do fuste por hectare aos 72 meses em função da área vital dos tratamentos e representação gráfica do modelo Exponencial ajustado; (b) Distribuição dos resíduos para volume por árvore em função do espaçamento ajustado com o modelo Exponencial, aos 72 meses; (c) Biomassa do fuste por hectare aos 72 meses em função da área vital dos tratamentos e representação gráfica do modelo Hiperbólico ajustado; (d) Distribuição dos resíduos para volume por árvore em função do espaçamento ajustado com o modelo Hiperbólico, aos 72 meses.

FIGURE 5: (a) Stem biomass per hectare in 72 months per vital area of the treatments and graphical representation of the Exponential model adjusted; (b) Distribution of the residues for the stem biomass per hectare based on the spacing adjusted with the Exponential model, at 72 months; (c) Stem biomass per hectare in 72 months per vital area of the treatments and graphical representation of the Hyperbolic model adjusted; (d) Distribution of the residues for the stem biomass per hectare based on the spacing adjusted with the Hyperbolic model, at 72 months.

do fuste por hectare, ocorreram nos intervalos entre $4,05 \mathrm{~m}^{2}$ a $4,95 \mathrm{~m}^{2}$, o que correspondeu a espaçamentos de $3 \times 1,35 \mathrm{~m}$ e $3 \times 1,65 \mathrm{~m}$, respectivamente.

Oliveira-Neto et al. (2003) trabalhando com Eucalyptus camaldulensis também observaram que a produção de biomassa por unidade de área decresceu com o aumento do espaçamento entre plantas. Segundo os autores, apesar do tamanho reduzido por árvore, a produção de biomassa de madeira, por unidade de área, aumentou com a redução do espaçamento, principalmente em razão do maior número de indivíduos por unidade de área. Goulart et al. (2003) também identificaram que a maior produção de biomassa do fuste por hectare foi influenciada pelo espaçamento, sendo que a biomassa diminuiu com o aumento do espaço vital. Os resultados obtidos neste estudo para biomassa do fuste não coincidem com os encontrados por Berger (2000) ao afirmar que a biomassa não sofreu influência do espaço vital.

\section{CONCLUSÕES}

A produção de biomassa do fuste por árvore aos 72 meses de idade foi afetada diretamente pelo espaçamento de plantio. As árvores de maiores dimensões ocorreram sistematicamente nos espaçamentos mais amplos, onde a competição por luz, água e nutrientes era menor entre as árvores devido à menor lotação por unidade de área. Diante deste aspecto, o espaçamento que resultou na maior produção de biomassa do fuste por árvore foi o de 3 x 5,25 m. 
Para os resultados da produção de biomassa do fuste por hectare foi observada uma resposta inversa ao aumento do espaçamento de plantio; nos espaçamentos adensados - tratamentos 1 a 4 - em que se tem maior número de árvores por hectare, houve também maior produção de biomassa do fuste por hectare, sendo o espaçamento $3 \times 0,50 \mathrm{~m}$ o que resultou na maior produção. Este resultado pode ser considerado como fator relevante na indústria de painéis de madeira, cujo objetivo é obter maior produção de biomassa por unidade de área. Espaçamentos adensados $-3 \times 0,50 \mathrm{~m}$ produzem em torno de $70 \mathrm{~m}^{3} / \mathrm{ha}$ a mais de biomassa do fuste por hectare do que espaçamentos amplos $-3 \times 5,25 \mathrm{~m}$; o que implica na possibilidade de redução da área de efetivo plantio para atender a uma mesma necessidade de abastecimento fabril, gerando benefícios econômicos para a empresa pelo fato dela poder reduzir o capital investido em terra e/ ou possibilidade de venda dela para geração de caixa.

Nos espaçamentos mais amplos foi maior a produção individual em volume e biomassa do fuste por árvore. Apesar de neste caso a produção por hectare diminuir, os ganhos em qualidade da madeira aumentam; pois há maior disponibilidade de área vital por planta, o que permite o desenvolvimento de árvores com maiores dimensões em diâmetro e altura e que tendem a reduzir os custos de colheita e implantação/manutenção da floresta. Na densidade básica da madeira, não houve aumento expressivo com o aumento da área vital a partir de 4,95 m²/árvore, ou seja, os acréscimos na densidade básica da madeira ocorreram do espaçamento $3 \times 0,50 \mathrm{~m}$ até o espaçamento $3 \times 1,65 \mathrm{~m}$ e a partir deste último não houve mais resposta significativa da densidade com o aumento do espaçamento. Diante deste aspecto é aceitável que, à medida que a competição entre as árvores foi reduzida em função do aumento do espaçamento, houve uma estagnação no aumento da densidade; mesmo aumentando o espaçamento não houve resposta positiva das árvores na variável densidade básica. Apesar de a densidade básica da madeira ter sido inferior nos espaçamentos $3 \times 0,50 \mathrm{~m}$ a $3 \times 0,90 \mathrm{~m}$, ela ainda permanecia crescente até atingir o seu máximo no espaçamento $3 \times 1,10 \mathrm{~m}$ e, a partir de então, tornou-se pouco variável, não sofrendo mais variações com o aumento da área vital por árvore.

\section{REFERÊNCIAS}

ASSIS, R. L. et al. Produção de biomassa de Eucalyptus urophylla S. T. Blake sob diferentes espaçamentos na região de cerrado de Minas Gerais. Revista Árvore, Viçosa, MG, v. 23, n. 2, p. 151-156, abr./jun. 1999. BALLONI, E. A. Fertilização florestal. Boletim Informativo IPEF, Piracicaba, n. 6, v. 16, n. A-1, p. 34, 1978.

ASSIS, R. L.; SIMÕES, J. W. Espaçamento de plantio e suas implicações silviculturais. Série Técnica IPEF, Piracicaba, v. 1, n. 3, p. 1-16, 1980.

BERGER, R. Crescimento e qualidade da madeira de um clone de Eucalyptus saligna Smith sob o efeito do espaçamento e da fertilização. 2000. 106 f. Dissertação (Mestrado) - Universidade Federal de Santa Maria, Santa Maria, 2000.

BERNARDO, A. L. Crescimento e eficiência nutricional de Eucalyptus spp. sob diferentes espaçamentos na região do cerrado de Minas Gerais. 1995. 102 f. Dissertação (Mestrado em Ciências Florestais) Universidade Federal de Viçosa, Viçosa, MG, 1995.

BOTELHO, S. A. Espaçamento. In: SCOLFORO, J. R. S. Manejo florestal. Lavras: UFLA/FAEPE, 1998. p. 381-405.

BRASIL, M. A. M.; FERREIRA, M. Variação da densidade básica da madeira de E. alba Reinw, E. saligna Smith e E. grandis Hill ex Maiden, aos 5 anos de idade, em função do local e do espaçamento. IPEF, Piracicaba, v. 2/3, p. 129-149, 1971.

CAMPOS, J. C. C.; LEITE, H. G. Mensuração florestal: perguntas e respostas. 2. ed. Viçosa, MG: UFV, 2006. $470 \mathrm{p}$.

COELHO, A. S. R.; MELLO, H. A.; SIMÕES, J. W. Comportamento de espécies de eucaliptos face ao espaçamento. IPEF, Piracicaba, n. 1, p. 29-55, 1970.

COUTO, L.; BRANDI, R. M.; CONDÉ, A. R. et al. Influência do espaçamento no crescimento do Eucalyptus urophylla, de origem híbrida, cultivado na região de Coronel Fabriciano, MG. Revista Árvore, Viçosa, MG, v. 1, n. 2, p. 57-71, 1977.

EVERT, F. Spacing studies: a review. Ottawa: Canadian Forestry Service, 1971. 95 p. (Information report, 
FMR-X-37).

FERREIRA, M. Estudo da variação da densidade básica da madeira de Eucalyptus alba Reiw e Eucalyptus saligna Smith. 1968. 71 f. Tese (Doutorado) - Escola Superior de Agricultura "Luiz de Queiroz", Piracicaba, 1968.

FERREIRA, M.; KAGEYAMA, P. Y. Melhoramento genético da densidade da madeira de eucalipto. IPEF, Piracicaba, v. 6, n. 20, supl, p. A1-A15, 1978.

FISHWICK, R. W. Estudos de espaçamentos e desbastes em plantações brasileiras. Brasil Florestal, Brasília, v. 7, n. 26, p. 13-23, 1976.

GARCIA, C. H.; CORRADINE, L.; ALVARENGA, S. F. Comportamento florestal do Eucalyptus grandis e Eucalyptus saligna em diferentes espaçamentos. Piracicaba: Instituto de Pesquisas e Estudos Florestais, 1991. 10 p. (Circular técnica, 179).

GOULART, M. et al. Massa específica básica e massa seca de madeira de Eucalyptus grandis sob efeito do espaçamento de plantio e da posição axial do tronco. Ciência Florestal, Santa Maria, v. 13, n. 2, p. $167-175,2003$.

JACOB, W. S.; BALLONI, E. A. Efeitos da fertilização na qualidade da madeira. Boletim Informativo IPEF, Piracicaba, v. 6, n .20, p. 1-12, 1978.

KELLISON, R. C.; RUSS, L.; FREDERICK, D. J. Effect os silvicultural practices on wood quality of southern hardwoods. Tappi Journal, Atlanta, v. 66, n. 1, p. 67-69, 1983.

LEVENE, H. In Contributions to probability and statistic: essays in honor of Harold Hotelling. Standford: Standford University Press, 1960. p. 278-292.

LIN, C. S.; MORSE, P. M. A compact design for spacing experiments. Biometrics, Washington, v. 31, p. 661-671, sept. 1975.

MELLO, H. A.; MASCARENHAS SOBRINHO, J.; SIMÕES, J. W. Influência do espaçamento na produção de madeira de eucalipto em solo de cerrado. IPEF, Piracicaba, v. 2/3, p. 3-30, 1971.

MELLO, H. A.; MASCARENHAS SOBRINHO, J.; SIMÕES, J. W. Influência do espaçamento e da idade de corte na produção de madeira de eucalipto em solo de cerrado. IPEF, v.13, p.143-62, 1976.

MISCHAN, M. M.; PINHO, S. Z. Experimentação agronômica: dados não balanceados. Botucatu: FUNDIBIO, 1996. 456 p.

NELDER, J. A. New kinds of systematic designs for spacing experiments. Biometrics, Washington, n. 18, p. 283-307, 1962.

OLIVEIRA-NETO, S. N. et al. Produção e distribuição de biomassa em Eucalyptus camaldulensis Dehn. em resposta à adubação e ao espaçamento. Revista Árvore, Viçosa, MG, v. 27, n. 1, p. 15-23, 2003.

PATIÑO-VALERA, F. Variação genética em progênies de Eucalyptus saligna Smith e sua interação com espaçamento. 1986. 192 f. Dissertação (Mestrado em Ciência Florestal) - Escola Superior de Agricultura Luiz de Queiroz, Universidade de São Paulo, Piracicaba, 1986.

PAULESKI, D. T. Influência do espaçamento sobre o crescimento e a qualidade da madeira de Pinus taeda L. 2010. 196 f. Tese (Doutorado em Ciência Florestal) - Universidade Federal de Santa Maria, Santa Maria, 2010.

PINKARD, E. A.; NEILSEN, W. A. Crown and stand characteristics of Eucalyptus nitens in response to initial spacing: implications for thinning. Forest Ecology and Management, Amsterdam, v. 172, n. 2/3, p. 215-227, jan. 2003.

REZENDE, M. A. Uma abordagem não convencional sobre as principais características físicas da madeira, com ênfase na retratibilidade, massa específica e técnica de atenuação da radiação gama. 1997. 138 f. Tese (Livre Docência em Agronomia/Energia na Agricultura) - Faculdade de Ciências Agronômicas da UNESP, Botucatu, 1997.

SANTOS, M. D. Efeito do espaçamento de plantio na biomassa do fuste de um clone híbrido interespecífico de Eucalyptus grandis e Eucalyptus urophylla. 2011. 140 f. Dissertação (Mestrado) Universidade Estadual Paulista Júlio de Mesquita Filho, Botucatu, 2011.

SCHNEIDER, P. S. P. Autodesbaste e diagrama de manejo da densidade em povoamentos de Pinus taeda L. 2008. 96 f. Dissertação (Mestrado) - Universidade Federal de Santa Maria, Santa Maria, 2008.

SCHÖNAU, A. P. G. The effect of planting espacement and pruning on growth, yield and timber density of Eucalyptus grandis. South African Forestry Journal, Pretoria, n. 88, p. 16-23, 1974. 
SCOLFORO, J. R. S. Biometria florestal: Parte I: modelos de regressão linear e não linear; Parte II: modelos para relação hipsométrica, volume, afilamento, e peso de matéria seca. Lavras: UFLA/FAEPE, 2005. $352 \mathrm{p}$.

SHIMOYAMA, V. R. S.; BARRICHELO, L. E. G. Densidade básica da madeira, melhoramento e manejo florestal. Série Técnica IPEF, Piracicaba, v. 6, n. 20, p. 1-22, ago. 1989.

SILVA, J. A. A.; MEUNIER, I. M. J; FERRAZ, I. Modelagem do crescimento em altura de Leucaena leucocephala (Lam.) de Wit., em solos do agreste de Pernambuco. Revista Árvore, Viçosa, MG, v. 20, n. 4, p. 443-450, 1996.

SOUZA, A. P.; DELlA LUCIA, R. M.; RESENDE, G. C. Estudo da densidade básica da madeira de Eucayptus microcorys F. Muell, cultivado na região de Dionísio, MG. Revista Árvore, Viçosa, MG, v. 3, n. 1, p. 16-27, jan./jun. 1979.

STAPE, J. L. Utilização de delineamento sistemático tipo leque no estudo de espaçamentos florestais. 1995. 86 f. Dissertação (Mestrado) - Escola Superior de Agricultura "Luiz de Queiroz", Piracicaba, 1995.

THIERSCH, C. R. Modelagem da densidade básica, do volume e do peso seco para plantios de clones de Eucalyptus sp. 2002. 197 f. Dissertação (Mestrado em Engenharia Florestal) - Universidade Federal de Lavras, Lavras, 2002.

TONINI, H. Crescimento e produção de clones Eucalyptus saligna Smith, na depressão central e serra do Sudeste, Rio Grande do Sul. 2003. 289 f. Tese (Doutorado em Ciências Florestais) - Universidade Federal de Santa Maria, Santa Maria, 2003.

VENUS, J. C.; CAUSTON, D. R. Plant growth analysis: the use of the Richards function as an alternative to polynomial exponentials. Annals of Botany, London, v. 43, p. 623-632, 1979.

VITAL, B. R.; DELLA LUCIA, R. M. Efeito do espaçamento na produção em peso e na qualidade da madeira de Eucalyptus grandis e Eucalyptus saligna aos 52 meses de idade. Revista Árvore, Viçosa, MG, v. 11, n. 2 , p. $132-145,1987$. 